\section{La sclérose en plaques et les médicaments immuno- modulateurs des récepteurs de la sphingosine 1-phosphate}

\author{
Franck Talmont, Anastassia Hatzoglou,
} Olivier Cuvillier

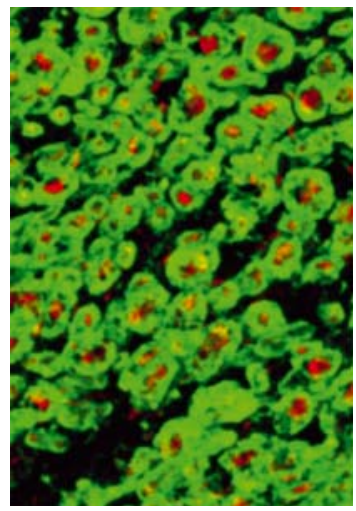

Institut de pharmacologie et de biologie structurale, UMR 5089 CNRS,

Université de Toulouse, 205 route de Narbonne, 31077 Toulouse, France. olivier.cuvillier@ipbs.fr franck.talmont@ipbs.fr

La maladie est caractérisée par l'apparition de handicaps variables tous dus à l'infiltration progressive de cellules du système immunitaire adaptatif, les lymphocytes $T$ et $B$, dans le système nerveux central (SNC). Ces cellules activées sont à l'origine de la destruction de la gaine de myéline des neurones et de l'apparition de dommages neuronaux et axonaux. On peut parfois observer la prolifération de cellules astrocytaires, ce qui peut conduire à des processus dichotomiques, en favorisant l'apparition de dommages supplémentaires, ou, au contraire, en contribuant aux phénomènes de réparation des lésions [3].

La SEP est une maladie auto-immune dont la progression est en relation directe avec le phénomène appelé « diversification antigénique » (epitope spreading). Dans plusieurs modèles murins de SEP, génétiques ou induits, la progression des dommages s'observe en effet en lien avec la présence de nouveaux épitopes qui sont reconnus par les anticorps produits par des lymphocytes B, et qui amplifie l'altération induite par les lymphocytes $T$ spécifiques de séquences peptidiques présentées par les neurones [4].

On distingue trois types de SEP. Une forme cyclique ou rémittente (relapsing-remitting multiple sclerosis, SEP-RR), qui touche $80-85 \%$ des patients, sans progression du handicap entre les poussées. Cette SEP peut évoluer en SEP secondairement progressive (ou SEP-SP). Pour plus de la moitié des malades, la SEP rémittente évolue vers la forme secondairement progressive en 15 à 20 ans [5]. Il existe également une

Vignette (Photo @ Inserm - Alice Lieury). 
forme primaire progressive (ou SEP-PP) atteignant environ $15 \%$ des patients $[1,2]$. Dans cette forme de SEP, les phases de la maladie ne sont pas entrecoupées de phases de rémission et la maladie progresse inexorablement. La transition entre la SEP rémittente et la forme progressive intervient lorsque les dommages axonaux ne sont plus compensés par les capacités reconstructrices du système nerveux central.

\section{Les symptômes}

Les symptômes de la SEP sont caractérisés par des phases aiguës de déficits neurologiques en récidive. Ils dépendent des régions du système nerveux central qui sont atteintes, et du niveau de démyélinisation et d'inflammation présenté par ces zones. Ainsi, lorsque les lésions inflammatoires affectent le nerf optique, les symptômes sont une déficience visuelle et une douleur oculaire lors des mouvements des yeux. Dans cette névrite optique, l'action des cytokines inflammatoires et de l'oxyde nitrique, au niveau des lésions nerveuses du nerf optique, sont les déterminants majeurs des symptômes [1]. Les axones démyélinisés peuvent ainsi devenir hyperexcitables et générer spontanément la perception de lumière clignotante.

C'est un ensemble de données cliniques, sérologiques et d'imagerie cérébrale qui permettent de poser le diagnostic. Pour la grande majorité des patients, la maladie débute par un épisode clinique isolé, régressant spontanément, qui implique le nerf optique, le tronc cérébral ou la moelle épinière. Les symptômes observés sont très hétérogènes et non spécifiques. Ils peuvent être uniques ou associés. On distingue différents symptômes cliniques [1]:

- Les troubles visuels (perception de flashs lumineux, oscillopsie, baisse de l'acuité, douleurs lors des mouvements oculaires).

- Les troubles moteurs (dystonie musculaire, altération de la mobilité, ataxie et tremblements, spasticité).

- Les troubles urinaires, intestinaux, sexuels.

- Les troubles de l'humeur (dépression et instabilité émotionnelle).

- Les troubles cognitifs (langage).

- Les troubles de la sensibilité.

- Les troubles de l'équilibre (vertiges).

Ces troubles sont souvent associés à une fatigue importante et à des douleurs (neuropathique, névralgie du nerf trijumeau, musculosquelettique, migraine).

En raison de la multiplicité des symptômes, il est essentiel de pouvoir éviter les erreurs de diagnostic qui peuvent avoir des conséquences importantes. Des critères de diagnostic, dits critères de McDonald, ont ainsi été développés et validés à partir des données cliniques de plusieurs centres académiques $[6,7]$. Validés sur une population d'adultes de moins de 50 ans d'origine génétique/ethnique européenne occidentale, les critères de McDonald peuvent néanmoins être utilisés pour des populations d'origines géographiques diverses, chez des enfants et des individus plus âgés. Les signes cliniques qui sont observés chez les patients sont confrontés aux résultats d'imagerie par résonance magnétique nucléaire (IRM) cérébrale et à une analyse du liquide céphalo-rachidien (LCR). L'analyse par IRM permet de rechercher la présence de lésions cérébrales, de définir leur localisation (dissémination spatiale), le moment de leur apparition (dissémination temporelle). Ces études d'imagerie cérébrale ont un rôle fondamental en terme de diagnostic, mais aussi pour le pronostic et l'évaluation de la réponse au traitement [8]. L'analyse du liquide céphalo-rachidien permet, elle, la recherche (en western blot) de bandes oligoclonales d'immunoglobulines. Elle représente un bon outil diagnostic de la SEP (avec une présence de ces bandes d'immunoglobulines observées chez plus de $95 \%$ des patients) qui permet de confirmer la sécrétion d'anticorps au niveau intra-thécal ${ }^{1}$.

\section{Les traitements (Tableau I)}

Le système immunitaire est responsable de la SEP et l'infiltration de cellules immunitaires dans le système nerveux central (SNC) est une caractéristique de toutes les formes de SEP. Les traitements disponibles permettent de modifier, de limiter ou d'inhiber la migration de ces cellules afin de réduire toute nouvelle agression. Actuellement, il n'existe en fait pas de traitement curatif de la SEP et les stratégies thérapeutiques visent à traiter les poussées, à mettre en place des traitements de fond et à traiter les symptômes associés. Les poussées, qui sont caractérisées par des symptômes apparaissant de manière soudaine, sont des signes pathologiques. Elles peuvent régresser spontanément plus ou moins complètement.

Ces crises inflammatoires, souvent très douloureuses, sont traitées à l'aide de perfusion de corticoïdes (Solumédrol ${ }^{\circledR}$ ) par voie intraveineuse (flash ou bolus de corticoïdes) $)^{2}$. Pour des attaques sévères au cours desquelles la corticothérapie s'est révélée être inefficace ou contre-indiquée, des échanges de plasma ont parfois été réalisés afin de modifier la composition plasmatique du patient. Les traitements de fond sont assurés par des molécules immunomodulatrices. Mais en cas d'échecs du traitement, et pour les formes agressives ou résistantes, des immunosuppresseurs seront utilisés. On distingue ainsi des traitements de $1^{\text {re }}, 2^{\mathrm{e}}$ et $3^{\mathrm{e}}$ ligne qui seront administrés selon l'évolution de la maladie.

\section{Les traitements de $1^{\text {re }}$ ligne}

- Les interférons bêta la et lb (Avonex ${ }^{\circledR}$, Pelgridy ${ }^{\circledR}$, Rebif ${ }^{\circledR}$, Betaferon ${ }^{\circledR}$, Extavia $\left.{ }^{\circledR}\right)$ stimulent l'activité de certaines cellules immunitaires et favorisent la production de cytokines immunosuppressives qui diminuent celle de cytokines pro-inflammatoires. Ces molécules sont administrées par voie sous-cutanée ou intramusculaire. Elles permettent de réduire le nombre et

\footnotetext{
${ }^{1}$ Ou sous-arachnoïdien.

${ }^{2}$ Le flash ou bolus correspond à une injection intraveineuse d'une importante quantité de corticoïdes en une seule dose.
} 


\begin{tabular}{|c|c|c|}
\hline $\begin{array}{l}\text { Médicament utilisé } \\
\text { en clinique }\end{array}$ & Cible/Mécanisme d'action & Effets secondaires indésirables \\
\hline Acétate de glatiramère & Immunomodulateur. & Réactions cutanées. \\
\hline Alemtuzumab & $\begin{array}{l}\text { Anticorps anti-CD20. Se lie aux cellules B et T. } \\
\text { Immunosuppresseur par lymphodéplétion. }\end{array}$ & $\begin{array}{l}\text { Cholécystite acalculeuse aiguë. } \\
\text { Réactions cardiovasculaires graves, hépatites } \\
\text { auto-immunes et lymphohistiocytoses } \\
\text { hémophagocytaires. }\end{array}$ \\
\hline Biotine & $\begin{array}{l}\text { Activation de l'acetylCoA carboxylase. } \\
\text { Néo-synthèse de myéline. }\end{array}$ & $\begin{array}{l}\text { Diarrhées, effet toxique sur la reproduction. } \\
\text { Incidence sur les résultats de tests biologiques } \\
\text { (fonctions thyroïdiennes et cardiaques, grossesse, } \\
\text { cortisol, sérologie VIH). }\end{array}$ \\
\hline Cladribine & $\begin{array}{l}\text { Cytotoxique immunomodulateur. Pro-drogue qui } \\
\text { après phosphorylation perturbe la synthèse et la } \\
\text { réparation de l'ADN. }\end{array}$ & $\begin{array}{l}\text { Réduction du nombre de lymphocytes, infections. } \\
\text { Risque potentiel de malignité. }\end{array}$ \\
\hline Corticostéroïdes & $\begin{array}{l}\text { Molécules et cellules de l'immunité. } \\
\text { Anti-inflammatoire et immunosuppresseur }\end{array}$ & $\begin{array}{l}\text { Troubles métaboliques, endocriniens, digestifs, } \\
\text { psychiques. } \\
\text { Aggravation d'états infectieux préexistants. }\end{array}$ \\
\hline Cyclophosphamide & $\begin{array}{l}\text { Immunosuppresseur. } \\
\text { Agent alkylant. }\end{array}$ & $\begin{array}{l}\text { Troubles digestifs. } \\
\text { Surveillance sanguine. }\end{array}$ \\
\hline Diméthyl fumarate & $\begin{array}{l}\text { Activation de la voie Nrf2 et augmentation de } \\
\text { l'expression de gènes antioxydants Nrf2-dépendants. } \\
\text { Protection des neurones vis-à-vis du stress oxydatif. }\end{array}$ & $\begin{array}{l}\text { Diarrhée, nausée, réduction du nombre de globules } \\
\text { blancs. }\end{array}$ \\
\hline Fingolimod & $\begin{array}{l}\text { Antagoniste fonctionnel des récepteurs de la } \\
\text { sphingosine l-phosphate. } \\
\text { Action par rétention lymphocytaire au niveau du } \\
\text { thymus et des ganglions lymphatiques. }\end{array}$ & $\begin{array}{l}\text { Bradycardie. } \\
\text { Redistribution des lymphocytes. }\end{array}$ \\
\hline Interféron Béta & $\begin{array}{l}\text { Récepteur IFN- } \alpha \text { de type } 1 . \\
\text { Inhibition de la production de cytokines Thl. } \\
\text { Immunomodulateur. } \\
\text { Anti-inflammatoire. }\end{array}$ & $\begin{array}{l}\text { Syndrome pseudo-grippal. } \\
\text { Réactions cutanées. } \\
\text { Atteinte du foie bénigne. }\end{array}$ \\
\hline Mitoxantrone & $\begin{array}{l}\text { Immunosuppresseur. } \\
\text { Agent intercalant. }\end{array}$ & $\begin{array}{l}\text { Leucémies aiguës myéloïdes pouvant être fatales, } \\
\text { insuffisance cardiaque congestive, aménorrhée, } \\
\text { toxicité sur l'ADN. }\end{array}$ \\
\hline Mycophénolate mofétil & $\begin{array}{l}\text { Immunosuppresseur. } \\
\text { Inhibiteur de la biosynthèse des purines. }\end{array}$ & $\begin{array}{l}\text { Troubles digestifs. } \\
\text { Surveillance sanguine. }\end{array}$ \\
\hline Natalizumab & $\begin{array}{l}\text { Anticorps anti-intégrine alpha } 4 \text { ( } \alpha 4 \beta 1 \text { et } \alpha 4 \beta 7 \text { ). } \\
\text { Permet de bloquer le passage des leucocytes } \\
\text { exprimant les intégrines } \alpha 4 \text { à travers la barrière } \\
\text { hémato-encéphalique. }\end{array}$ & $\begin{array}{l}\text { Infection urinaire, urticaire, céphalées. } \\
\text { Augmente le risque de leucoencéphalopathie } \\
\text { multifocale progressive. }\end{array}$ \\
\hline Ocrelizumab & $\begin{array}{l}\text { Anticorps anti-CD20. Se lie aux cellules B adultes et } \\
\text { mémoire. } \\
\text { Immunomodulateur par lymphodéplétion. }\end{array}$ & $\begin{array}{l}\text { Irritation au site d'injection, fièvre, nausée, } \\
\text { abaissement de la pression artérielle. Risque de } \\
\text { cancers. }\end{array}$ \\
\hline Tériflunomide & $\begin{array}{l}\text { Immunomodulateur de la prolifération des } \\
\text { lymphocytes par inhibition de la synthèse de la } \\
\text { pyrimidine. Inhibition de l'activation et de la } \\
\text { sécrétion des cytokines et de l'interféron gamma. }\end{array}$ & $\begin{array}{l}\text { Symptômes digestifs, rétrécissement capillaire. } \\
\text { Lymphopénie sévère. }\end{array}$ \\
\hline
\end{tabular}

Tableau I. Médicaments utilisés dans le cadre du traitement de la SEP. 
l'intensité des poussées. Elles ont cependant le désavantage d'entraîner l'apparition d'un syndrome pseudogrippal et des réactions cutanées après l'injection.

- L'acétate de glatiramère (Copaxone ${ }^{\circledR}$ ) est un mélange de peptides de tailles aléatoires (40 à 100 résidus) qui sont constitués d'acides aminés (acide glutamique, lysine, alanine, et tyrosine) qui composent la MBP (myelin basic protein), l'un des constituants de la myéline. Ces acides aminés altéreraient la présentation des antigènes produits à partir de la myéline aux lymphocytes T auto-réactifs [9]. Ils auraient également la capacité d'induire la production de cytokines anti-inflammatoires.

- Le diméthylfumarate (Tecfidera ${ }^{\circledR}$ ) induit la production de cytokines anti-inflammatoires et la prolifération de cellules dendritiques. II améliore la protection des neurones contre le stress oxydatif, et favorise la mort par apoptose des lymphocytes T activés.

- Le tériflunomide (Aubagio ${ }^{\circledR}$ ) agit en inhibant la prolifération des lymphocytes. Il peut néanmoins provoquer des dommages hépatiques importants.

\section{Traitements de $2^{\mathrm{e}}$ ligne}

- L'ocrelizumab (0crevus ${ }^{\circledR}$ ) est un anticorps anti-CD20 humanisé recombinant. La liaison de cet anticorps à la large boucle extracellulaire de la protéine CD20, exprimée par les lymphocytes $B$, est à l'origine de la déplétion des lymphocytes $B \mathrm{CD} 20^{+}$. Cet anticorps agit par apoptose, en induisant une cytotoxicité dépendante du complément, une phagocytose et une cytotoxicité à médiation cellulaire dépendante des anticorps. Les lymphocytes $B C^{2} 20^{+}$sont impliqués dans la sécrétion des cytokines proinflammatoires, dans la présentation des auto-antigènes aux lymphocytes $T$ et dans la sécrétion des auto-anticorps. La déplétion sélective des lymphocytes $B C D 20^{+}$a ainsi un effet bénéfique sur la SEP rémittente, mais également sur la SEP progressive primaire [10].

- L'anticorps monoclonal natalizumab (Tysabri®) est un anticorps dirigé contre la sousunité $\alpha 4$ des intégrines $\alpha 4 \beta 1$ [11] $(\rightarrow)$ et $\alpha 4 \beta 7$. Il permet de bloquer la migration des

$(\rightarrow)$ Voir la Synthèse de C. Papeix et C. Lubetzki, $\mathrm{m} / \mathrm{s} \mathrm{n}^{\circ} 12$, décembre 2009 , page 1113 lymphocytes $T$ à travers la barrière hématoencéphalique et d'atteindre le système nerveux central. II peut, cependant, induire la réactivation du virus JC, un papovavirus qui provoque une leucoencéphalopathie multifocale . $^{3}$.

- Le fingolimod (Gilenya ${ }^{\circledR}$, FTY720) agit sur les récepteurs de la sphingosine 1-phosphate (SIP) qui sont exprimés par les lymphocytes T. II permet de séquestrer les lymphocytes $T$ activés, leur interdisant de rejoindre les foyers inflammatoires. Une surveillance cardiovasculaire doit cependant être pratiquée.

Comparé à un traitement initial par l'acétate de glatiramère ou l'interféron bêta, un traitement par le fingolimod, l'alemtuzumab (Lemtrada ${ }^{\circledR}$ ), un anticorps humanisé recombinant dirigé contre la molécule CD52 exprimée à la surface des lymphocytes B et T, qui induit leur déplétion par cytotoxicité cellulaire dépendante d'anticorps et/ ou du complément, ou le natalizumab est associé à une réduction du risque de conversion vers la SEP secondairement progressive [12].

${ }^{3}$ C'est aussi le cas de l'anti-CD20, l'ocrelizumab.
- La cladribine (Mavenclad ${ }^{\circledR}$ ) est une prodrogue, analogue nucléosidique de la désoxyadénosine, qui interfère dans la synthèse et la réparation de l'ADN. Cette molécule, active sous forme phosphorylée, entraîne la déplétion sélective des lymphocytes B et T [13].

\section{Traitements de $3^{\mathrm{e}}$ ligne}

- L'alemtuzumab présenté ci-dessus est utilisé en traitement de $3^{e}$ ligne. La liaison de l'anticorps à la glycoprotéine CD52 provoque la lyse des lymphocytes B et T via le complément et une cytotoxicité à médiation cellulaire dépendante de l'anticorps. Cet anticorps induit donc une déplétion lymphocytaire mais, en raison d'effets secondaires pouvant se révéler très importants, ce traitement est réservé aux patients adultes atteints de SEP cyclique très active et réfractaire aux autres traitements de fond [14].

\section{Les traitements par immunosuppresseurs}

La mitoxantrone (Novantrone ${ }^{\circledR}$ ) est un agent intercalant de I'ADN et un inhibiteur de la topoisomérase II. Elle est utilisée pour traiter les formes très sévères de SEP, mais elle présente des effets secondaires très importants, car associés au développement de cancers du sang. Le cyclophosphamide (Endoxan ${ }^{\circledR}$ ) et le mycophenolate mofétil ( $\left(\varepsilon l\right.$ cept ${ }^{\circledR}$ ) sont également des immunosuppresseurs (hors autorisation de mise sur le marché dans cette indication) qui permettent, dans une certaine mesure, de stabiliser la maladie dans le temps. Tous deux présentent des effets secondaires défavorables, en particulier digestifs, et nécessitent une surveillance sanguine.

\section{Les traitements des symptômes associés}

Ces traitements sont nécessaires pour améliorer la qualité de vie des patients. Ils incluent des médicaments antidouleurs, myorelaxants, antidépresseurs, antifatigues, anticonvulsants, laxatifs, antidiarrhéiques, des médicaments pour traiter les troubles urinaires, intestinaux et sexuels. Ils peuvent être associés à des kinésithérapies, des thérapies comportementales, de la méditation, etc.

\section{Le fingolimod, la sphingosine 1-phosphate et la SEP}

Depuis la mise sur le marché du fingolimod (FTY720) en 2010 et 2011, par les agences de médecine américaine et européenne, la famille regroupant les cinq récepteurs de la sphingosine 1-phosphate $\left(S 1 P R_{1-5}\right)$ (Figure 1) est devenue une cible thérapeutique de choix dans le traitement de la SEP. Le fingolimod est un dérivé de la myriocine (Figure 2), une molécule 


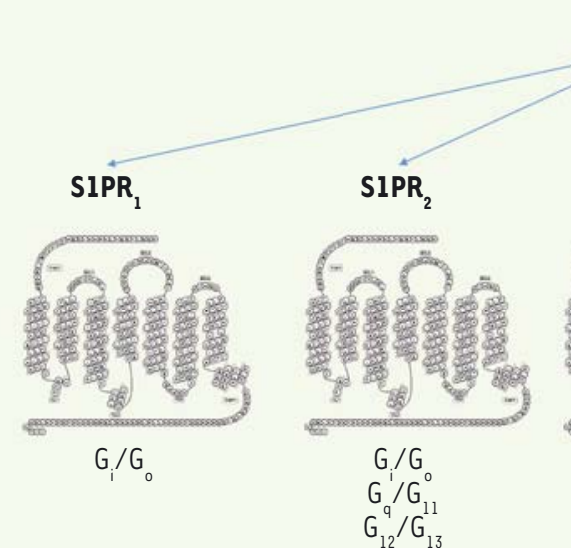

Fingolimod Siponimod Ozanimod Ponesimod Amiselimod GSK2018682 Céralifimod
SIP

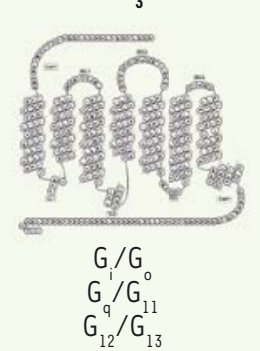

Fingolimod

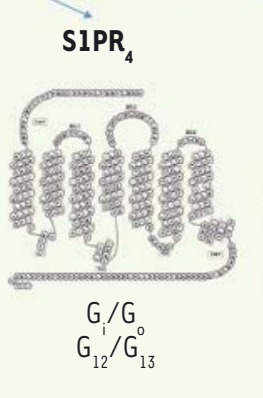

Fingolimod

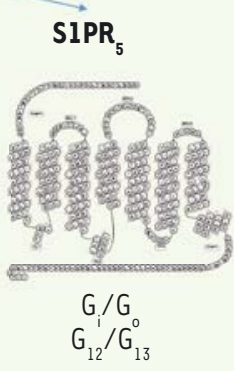

Fingolimod Siponimod Ozanimod Amiselimod GSK2018682 Céralifimod

Figure 1. Activation des récepteurs de la sphingosine 1-phosphate par la sphingosine 1-phosphate et par les médicaments qui les ciblent.

immunosuppressive extraite du champignon Isaria sinclairii utilisé dans la pharmacopée chinoise. Cette molécule a d'abord été utilisée dans le cadre de rejets aigus de greffe de rein, mais sans dépasser les essais cliniques de phase III pour cette indication, en raison d'absence d'effets thérapeutiques supérieurs à la thérapie de référence, et de la survenue d'effets secondaires délétères. Dans la SEP, le fingolimod est utilisé à des doses dix fois inférieures à celles utilisées en transplantation rénale, ce qui réduit les risques d'effets secondaires. II permet de diminuer le nombre de rechutes cliniques et augmente les délais d'apparition des évènements conduisant à l'invalidité dans les formes récurrentes de SEP. Dans les formes primaires progressives, le fingolimod n'a néanmoins pas montré d'avantages par rapport à un placebo [15].

II s'agit d'une prodrogue qui est phosphorylée par la sphingosine kinase 2 (SphK2) en fingolimod-P ou FTY720-P, une molécule qui mime les effets d'un médiateur lipidique naturel, la sphingosine-1-phosphate (SIP). Immunomodulateur, son principal mode d'action repose sur son effet sur le trafic des lymphocytes [13] : il favorise leur rétention dans les organes lymphoïdes primaires et secondaires. L'inhibition de l'infiltration des lymphocytes dans le système nerveux central est ainsi une conséquence de leur rétention. II agit en redistribuant de manière réversible les lymphocytes $T$ et $B$ de la circulation dans les organes lymphoïdes secondaires, tels que les ganglions lymphatiques périphériques et mésentériques et les plaques de Peyer (au niveau intestinal), provoquant un état de lymphopénie périphérique. Cette quasi absence de lymphocytes dans la circulation périphérique peut cependant avoir une conséquence délétère : le développement d'infections microbiennes opportunistes. Néanmoins, dans ce cas, l'arrêt du traitement permet une réactivation immédiate de la réponse immunitaire adaptative. La lymphopénie induite par le fingolimod ne cor- respond donc pas à une perte générale de lymphocytes dans tout l'organisme, contrairement à la véritable lymphopénie induite par irradiation ou traitement par l'alemtuzumab pour laquelle la restauration de l'immunité lymphocytaire nécessite une reconstitution du système immunitaire à partir des précurseurs cellulaires de la moelle osseuse.

\section{Le fingolimod, la sphingosine 1-phosphate et les récepteurs de la sphingosine 1-phosphate}

Structuralement, une fois phosphorylé, le fingolimod est un analogue de la sphingosine 1-phosphate (S1P) (Figure 2).

La principale source de SIP est constituée par les érythrocytes et les cellules endothéliales [16]. Dans le sang, elle est véhiculée sous forme combinée à des protéines de transport, telles que les HDL (high-density lipoprotein, $60 \%)$ et l'albumine ( $30 \%)$, et dans une moindre mesure aux VLDL (very low-density lipoprotein) et aux LDL (low-density lipoprotein) [17]. Sa liaison aux HDL se réalise via l'apolipoprotéine ApoM qui agit comme une protéine chaperonne, contrôlant ainsi les niveaux de SIP dans le sang. Sa concentration est de l'ordre du micromolaire dans le plasma. Elle est moins importante dans les liquides des compartiments tissulaires, ce qui explique le gradient de concentration existant entre le sang et les tissus. La SPI se lie, avec de fortes affinités (nanomolaires) à la famille de récepteurs de la SIP, initialement nommée famille de gènes 


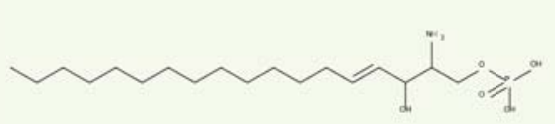

Sphingosine 1-phosphate
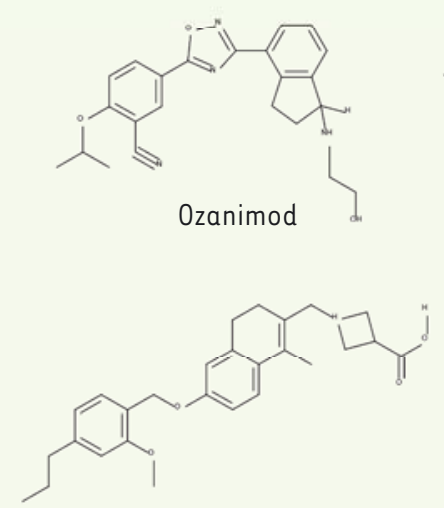

Ceralifimod

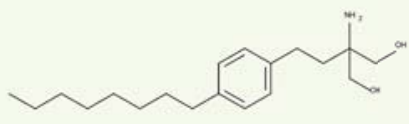

Fingolimod

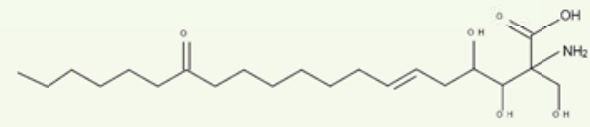

Myriocine

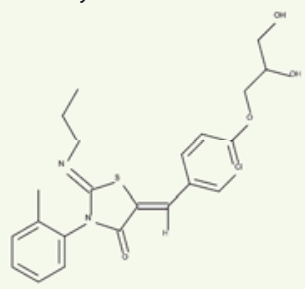

Ponesimod
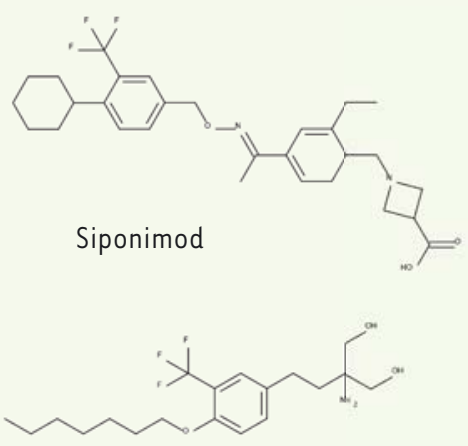

Amiselimod

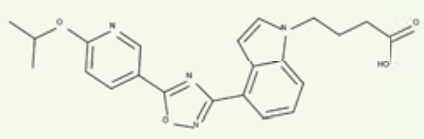

GSK-2018682

Figure 2. Structure de la sphingosine 1-phosphate et des médicaments ciblant les récepteurs de la sphingosine 1-phosphate et utilisés dans le traitement de la SEP.

de différenciation endothéliale (EDG) des récepteurs lipidiques [18], qui compte cinq membres: $\mathrm{SIPR}_{1-5}$ (Figure 1 ). Ces cinq récepteurs partagent des similitudes de séquence et de structure génomique. Ils appartiennent à la superfamille des récepteurs couplés aux protéines $G(R C P G)$ qui sont impliqués dans de nombreux processus biologiques clés, tels que l'odorat, le goût et la vision [19]. Selon le séquençage du génome humain, $60 \%$ des 800 RCPG identifiés appartiennent aux récepteurs dits olfactifs ou sensoriels. Les $40 \%$ restants sont classés dans les cinq familles principales du système GRAFS (glutamate, rhodopsine, adhésion, frizzled/taste2 et secrétine) [20]. Compte tenu de leur rôle dans de nombreux processus physiologiques et pathologiques, les RCPG sont devenus des cibles de choix pour le développement de nouveaux médicaments. Près de la moitié des médicaments commercialisés par l'industrie pharmaceutique ciblent en effet cette classe de récepteurs. Les récepteurs de la SIP font partie de la famille de la rhodopsine et sont couplés à différentes protéines G (Figure 1). Leur liaison à la SIP induit l'activation de voies de signalisation variées impliquées dans les phénomènes de survie, croissance, migration et différenciation cellulaire $[21](\rightarrow)$.

$(\rightarrow)$ Voir la Synthèse de 0 . Cuvillier, $m / s n^{\circ} 11$, novembre 2012, page 951

Le récepteur $S I P R_{1}$ a été initialement détecté dans les cellules endothéliales de la veine ombilicale (HUVEC) transformées par le phorbol 12-myristate 13-acétate (PMA). La voie de signalisation du S1PR comprend le couplage à la famille de protéines Gi/o et donc l'inhibition de l'adénylate cyclase (ou adénylyl cyclase), l'activation de la phosphoinositide 3-kinase et de la phospholipase C. Chez la souris, l'analyse des transcrits codant $S 1 P R_{1}$ montre que ce récepteur est fortement exprimé dans le tissu adipeux, la rate, le poumon, le cerveau, le foie et le cœur, et faiblement dans le muscle squelettique, le thymus, l'utérus et les reins [22]. La délétion du gène $\mathrm{SIpr}_{1}$ dans la lignée germinale de souris entraîne la létalité in utero [23]. En fait, le récepteur $\mathrm{SIPR}_{1}$ joue un rôle vital dans le développement vasculaire et la létalité chez la souris est due à un défaut du développement des vaisseaux sanguins. $S 1 P R_{1}$ a également une fonction essentielle dans la migration cellulaire, en particulier dans le drainage des lymphocytes $T$ du thymus vers le sang et les structures lymphoïdes environnantes [24].

Plusieurs études ont révélé l'expression des récepteurs SIPR dans le système nerveux central [25], plus précisément à la surface des astrocytes chez l'homme, et dans l'endothélium chez l'homme et les rongeurs. Les récepteurs SIPR $2-4$ seraient, quant à eux, largement distribués et l'expression de $\mathrm{SIPR}_{5}$ serait restreinte au cerveau et aux lymphocytes $T$. La localisation de ces récepteurs reste toutefois difficile à déterminer, la production d'anticorps spécifiques n'étant pas aisée [26-28].

$\mathrm{SIPR}_{2}$ auquel ne se lie pas le fingolimod, est exprimé en particulier par les macrophages et les cellules progénitrices d'oligodendrocytes [29]. Chez la souris, l'inactivation du gène $\mathrm{SIpr}_{2}$ permet de réduire la démyélinisation et d'augmenter la remyélinisation dans un modèle murin de SEP [30]. Cet effet très positif est cependant contrebalancé par l'apparition de lymphomes à lymphocytes $B$ [31]. 
$\mathrm{SlPR}_{5}$ est principalement exprimé dans la substance blanche tout au long du développement de la lignée des oligodendrocytes et dans les oligodendrocytes matures [32]. Il est aussi présent au niveau des capillaires cérébraux et son rôle dans la fonction de barrière endothéliale du cerveau a été suggéré [33]. La signalisation induite par SIPR comprend le couplage à des protéines $G_{i / 0}$ et $G_{12 / 13}$.

\section{Mécanisme d'action du fingolimod}

Le traitement thérapeutique qui repose sur le fingolimod est associé à sa phosphorylation in vivo par la sphingosine kinase-2 qui produira le fingolimod-P (FTY720-P). Contrairement à la S1P, le fingolimod est principalement transporté dans le sang en association avec l'albumine. Le FTY720-P est un véritable agoniste car il a la capacité d'activer ses récepteurs et d'induire une cascade d'évènements cellulaires. C'est aussi un antagoniste fonctionnel. En effet, après sa liaison aux récepteurs présents à la surface des lymphocytes, le FTY720-P induit l'internalisation des récepteurs et leur dégradation dans les compartiments intracellulaires [34], dégradation qui ne serait pas compensée par leur néosynthèse. Le FTY720-P est un puissant activateur de l'ubiquitination du récepteur $S I P R_{1}$. Cette modification, mise en évidence dans une étude réalisée sur les cellules 293 de rein embryonnaire humain (HEK) [35], entraîne une dégradation rapide et quantitative du récepteur. La capacité des agonistes pharmacologiques à induire la dégradation de $\mathrm{SIPR}_{1}$, mais aussi des autres récepteurs, pourrait ainsi expliquer leurs propriétés immunosuppressives : après leur internalisation et leur dégradation, leur absence de la membrane conduirait à l'impossibilité pour les lymphocytes activés de «sentir » le gradient de SIP et donc à leur séquestration dans le thymus et les organes lymphoïdes secondaires. L'activation des récepteurs par le FTY720-P permettrait également d'accroître le rôle de barrière des cellules endothéliales [36]. Bien que qualifiées de non spécifiques car activées par des concentrations très importantes de produit, de l'ordre du $\mu \mathrm{M}$, des cibles intracellulaires ont aussi été décrites pour le fingolimod et le fingolimod-P. Le fingolimod inhibe en effet des enzymes clés du métabolisme du céramide [36], modulant ainsi les effets pro-inflammatoires de cette classe de molécules dans le cadre du traitement de la SEP.

\section{Les modulateurs des SIPR de deuxième génération}

Les effets secondaires [36] occasionnés par l'administration journalière de fingolimod ont incité l'industrie pharmaceutique à développer des principes actifs possédant un meilleur profil de sécurité, une meilleure sélectivité et une durée de vie pharmacocinétique réduite. Le fingolimod est associé à une diminution transitoire de la fréquence cardiaque (brady-arythmie) ainsi qu'à des retards de conduction auriculo-ventriculaire. De même, dans un modèle animal et sur cœur perfusé [37], il a été montré que le récepteur $\mathrm{SIPR}_{3}$ était impliqué dans la régulation du rythme cardiaque. Des effets indésirables cardiaques, dont des décès ont ainsi été rapportés chez des patients présentant déjà une maladie cardiaque. Des contre-indications ont donc été émises pour les patients ayant présenté, entre autres, un infarctus du myocarde ou une arythmie cardiaque sévère.
Des molécules ayant une meilleure sélectivité que le fingolimod tout en gardant une voie d'administration orale ont été développées (Figure 2). Le ponesimod et l'amiselimod ciblent principalement les récepteurs $\mathrm{SIPR}_{1}$. D'autres molécules ciblent à la fois les récepteurs $\mathrm{SIPR}_{1}$ et $S 1 P R_{5}$, telles le siponimod, l'ozanimod, le ceralifimod, le GSK2018682. Ces modulateurs ne ciblant pas $\mathrm{SIPR}_{3}$, les effets secondaires cardiaques pourraient être évités. L'activation de $S_{1 P R_{5}}$ aurait également un effet neuroprotecteur sur les oligodendrocytes. Certaines de ces molécules ont une activité immunomodulatrice intrinsèque qui ne nécessite pas de phosphorylation in vivo comme le fingolimod, ce qui permet d'éviter les effets éventuels hors du contexte des récepteurs que présente le fingolimod. Le siponimod (Mayzent ${ }^{\circledR}$ ) vient ainsi d'être autorisé par la Food and drug administration (FDA) américaine, dans le cadre du traitement de la SEP secondairement progressive, en plus de ses indications dans la SEP rémittente [5]. Une analyse de progression d'incapacité à 3 mois révèle que ce médicament permet de réduire de façon significative le risque de progression de la maladie.

\section{Vers des médicaments curatifs de la SEP}

\section{Remyéliniser les axones}

La démyélinisation et les lésions axonales ont un rôle critique dans la survenue des déficits neurologiques des patients atteints de SEP. Un processus naturel permet la remyélinisation après l'apparition des dommages axonaux. Malheureusement, ce processus ne permet pas de compenser les atteintes dues à la progression des attaques auto-immunes. Les voies de recherche curative se focalisent donc sur des éléments qui permettraient de stopper la démyélinisation ou d'aider à la réparation ou à la régénération de la gaine de myéline [38].

Des travaux sont ainsi en cours concernant le rôle des histones déacétylases (HDAC) dans la myélinisation des axones. La délétion du gène codant une HDAC (I'HDACll) permet d'améliorer les symptômes cliniques dans l'encéphalomyélite auto-immune expérimentale $(\varepsilon A \varepsilon)$, un modèle murin de SEP [39]. L'inhibition de l'expression de cette protéine aurait un effet positif sur la différenciation des cellules précurseurs d'oligodendrocytes et des cellules souches neuronales et donc aiderait à la reconstruction de la gaine de myéline.

$L^{\prime} o p i c i n u m a b{ }^{\circledR}$, un anticorps monoclonal spécifique de Lingo-1 (leucine-rich repeat and immunoglobulin-like domain-containing protein 1), une protéine extrêmement conservée présente au niveau de la gaine de myéline, a été testé [40]. Cet anticorps, qui inhibe Lingo-1, stimule la prolifération et la différenciation 
des cellules précurseurs d'oligodendrocytes que cette protéine bloque [40]. Chez l'animal, après administration de cet anticorps, on observe une moindre destruction des gaines de myéline, une meilleure préservation des fibres nerveuses, une atténuation des signes cliniques, et une remyélinisation plus efficace. Des essais cliniques ont été réalisés et une amélioration de la conduction du nerf optique a été observée dans la névrite optique [41]. Malheureusement, aucun effet n'a été obtenu dans la SEP rémittente [42]. Les essais cliniques se poursuivent en introduisant des critères d'inclusion mieux adaptés.

La clémastine, un antagoniste antihistaminique des récepteurs $\mathrm{Hl}$ de l'histamine, a également montré des effets remyélinisants dans des modèles expérimentaux animaux. Chez des patients atteints de neuropathie optique chronique, on a pu observer une amélioration de leur état et des essais cliniques sont toujours en cours [43].

Les SIPR sont exprimés par de nombreuses cellules impliquées dans la myélinisation (cellules microgliales, oligodentrocytes, cellules progénitrices d'oligodendrocytes) [44]. Dans un modèle in vitro de démyélinisation induite chimiquement sur des cultures de sphéroïdes de télencéphale de rat, l'activation des SIPR par le fingolimod ou le siponimod permet d'augmenter l'expression des marqueurs de remyélinisation [45]. In vivo, dans un modèle murin, le fingolimod favorise la régénération nerveuse du nerf sciatique et, ex vivo, il stimule la production de myéline [46]. Cet effet de stimulation n'a cependant pas été confirmé dans le modèle animal d'induction de démyélinisation par la cuprizone [47], bien qu'un effet neuroprotecteur ait été observé, y compris avec le siponimod [48]. Les SIPR et leurs modulateurs, en développement, représentent ainsi un champ d'étude très intéressant dans un contexte protecteur, mais également stimulateur de myélinisation.

La biotine (ou vitamine B7) à haute dose, c'est-à-dire à des concentrations mille fois supérieures à celles recommandées pour un sujet sain, permet de réduire les symptômes des SEP progressives primaires et secondaires. La biotine activerait des enzymes impliqués dans la biosynthèse de la myéline et dans la production d'énergie dans les axones démyélinisés [49]. Elle est actuellement délivrée par les pharmacies hospitalières à des patients déjà traités en attendant une demande formelle d'autorisation de mise sur le marché.

\section{Les thérapies fondées sur les cellules souches}

Initié il y a déjà une vingtaine d'années, le traitement de la SEP à l'aide de cellules souches reste une voie encore prometteuse. Deux procédures ont été décrites [50].

La première fait appel à une immuno-ablation chimique à l'aide de cyclophosphamide et de rATG (une préparation d'immunoglobulines anti-thymocytes) $[51,52]$ suivie d'une transplantation autologue de cellules souches hématopoïétiques (aHSC). L'immuno-ablation par irradiation du corps entier n'est plus réalisée, en raison de toxicités sévères. Cette thérapie immuno-ablative, suivie d'une thérapie immuno-reconstitutive à l'aide de cellules hématopoïétiques, est considérée comme permettant au système immunitaire du patient de reprendre un état pré-pathologique. L'entrée en phase aplasique, caractérisée par un taux extrêmement bas de cellules hématopoïétiques dans le sang, nécessite l'administration en parallèle de traitements antiviraux et antibacté- riens. De nombreux essais cliniques ont été réalisés chez des patients présentant une SEP très active et réfractaire aux traitements pour lesquels cette thérapie a permis d'induire des rémissions et une amélioration de leur état [51-53]. L'efficacité et la sûreté des traitements sur le long terme nécessitent cependant encore d'être évaluées.

La deuxième thérapie est également une thérapie cellulaire. Elle repose sur l'injection aux patients de cellules multipotentes possédant à la fois des propriétés immunomodulatrices et régénératrices. Des études précliniques ont été réalisées à l'aide de cellules souches mésenchymateuses (MSC), neuronales (NSC), embryonnaires (hESC) ou pluripotentes inductibles (iPSC) [50]. Dans le modèle de souris présentant une encéphalomyélite auto-immune expérimentale ( $(A \varepsilon)$, l'injection de MSC conduit à la diminution de l'infiltration des cellules immunitaires, de la démyélinisation et des dommages axonaux. Chez l'homme, de nombreuses études, réalisées dans des cohortes réduites, ont permis d'explorer la faisabilité et la sécurité de la transplantation de ces cellules. Leur injection à des patients s'est révélée avoir un bon profil de sécurité, mais une efficacité très variable [54]. Un essai clinique multicentrique de phase I/II, appelé MESEMS (mesenchymal stem cell for multiple sclerosis) actuellement en cours [55] devrait permettre d'évaluer avec certitude le profil de sécurité et l'efficacité de ce traitement. Les autres types de cellules souches sont l'objet de recherches qui sont essentiellement au stade préclinique avec quelques essais de sécurité chez l'homme [56]. Si peu d'effets secondaires défavorables ont été observés avec ces thérapies cellulaires, il existe néanmoins, sur le long terme, un risque non négligeable de transformation maligne.

Les voies de recherche de traitements palliatifs et curatifs de la SEP sont prometteuses. Les causes de la démyélinisation sont multiples. Elles reposent sur les lymphocytes $T$, les lymphocytes $B$ et la sécrétion d'anticorps, ainsi que sur la production de cytokines. La connaissance des mécanismes à l'origine de l'infiltration anormale des cellules immunitaires à travers la barrière immuno-encéphalique reste essentielle. La compréhension globale des phénomènes inflammatoires et immunitaires participant à la pathogenèse de la SEP permettra ainsi de traiter de manière durable et peutêtre d'éviter l'apparition de la maladie. $\diamond$

\section{SUMMARY}

Multiple sclerosis and immuno-modulators of sphingosine $\mathbf{l}$-phosphate receptors

Multiple sclerosis (MS) is a disease of the central nervous system with a very debilitating inflammatory component that usually affects young people (years 20-40). 
This disease is characterized by the progressive destruction of the myelin sheath of the axons by the cells of the immune system, which results in neuronal degeneration. The $T$ and $B$ lymphocytes are the main players in this disease, which can be remittent with relapses or progressive. Among the drugs used to treat MS is the immunosuppressor fingolimod, the targets of which are the sphingosine 1-phosphate receptors. This molecule acts orally by preventing lymphocytes from leaving the thymus and lymph nodes and from reaching inflammatory brain foci. Other immunosuppressive drugs affecting sphingosine 1-phosphate receptors are under development and an intense search for curative drugs and treatments is being conducted. $\diamond$

\section{LIENS D'INTÉRÊT}

Les auteurs déclarent n'avoir aucun lien d'intérêt concernant les données publiées dans cet article.

\section{RÉFÉRENCES}

1. Thompson AJ, Baranzini SE, Geurts J, et al. Multiple sclerosis. Lancet 2018 ; $391: 1622-36$.

2. Zephir H. Progress in understanding the pathophysiology of multiple sclerosis. Rev Neurol (Paris) $2018 ; 174: 358-63$.

3. Brambilla R. The contribution of astrocytes to the neuroinflammatory response in multiple sclerosis and experimental autoimmune encephalomyelitis. Acta Neuropathol 2019 ; 137 : 757-83.

4. Cornaby C, Gibbons L, Mayhew V, et al. B cell epitope spreading: mechanisms and contribution to autoimmune diseases. Immunol Lett $2015 ; 163: 56-68$.

5. Kappos L, Bar-Or A, Cree BAC, et al. Siponimod versus placebo in secondary progressive multiple sclerosis (EXPAND): a double-blind, randomised, phase 3 study. Lancet 2018 ; 391 : 1263-73.

6. Polman CH, Reingold SC, Banwell B, et al. Diagnostic criteria for multiple sclerosis: 2010 revisions to the McDonald criteria. Ann Neurol $2011 ; 69: 292-302$.

7. Thompson AJ, Banwell BL, Barkhof F, et al. Diagnosis of multiple sclerosis: 2017 revisions of the McDonald criteria. Lancet Neurol 2018 ; 17 : 162-73.

8. Cortese R, Collorone S, Ciccarelli 0 , Toosy AT. Advances in brain imaging in multiple sclerosis. Ther Adv Neurol Disord 2019; 12 : 1756286419859722.

9. Weber MS, Hohlfeld R, Zamvil SS. Mechanism of action of glatiramer acetate in treatment of multiple sclerosis. Neurotherapeutics $2007 ; 4: 647-53$.

10. Gelfand JM, Cree BAC, Hauser SL. Ocrelizumab and other CD20+ B-cell-depleting therapies in multiple sclerosis. Neurotherapeutics $2017 ; 14: 835-41$.

11. Papeix C, Lubetzki C. Anticorps monoclonaux dans la sclérose en plaques. Med Sci (Paris) 2009; $25: 1113-5$.

12. Brown JWL, Coles A, Horakova D, et al. Association of initial disease-modifying therapy with later conversion to secondary progressive multiple sclerosis. JAMA 2019 ; $321: 175-87$.

13. Rommer PS, Milo R, Han MH, et al. Immunological aspects of approved MS therapeutics. Front Immunol $2019 ; 10: 1564$.

14. Holmoy T, Fevang B, Olsen DB, et al. Adverse events with fatal outcome associated with alemtuzumab treatment in multiple sclerosis. BMC Res Notes $2019 ; 12: 497$.

15. Lublin F, Miller DH, Freedman MS, et al. Oral fingolimod in primary progressive multiple sclerosis (INFORMS): a phase 3, randomised, double-blind, placebo-controlled trial. Lancet 2016; 387 : 1075-84.

16. Gazit SL, Mariko B, Therond P, et al. Platelet and erythrocyte sources of SIP are redundant for vascular development and homeostasis, but both rendered essential after plasma SIP depletion in anaphylactic shock. Circ Res $2016 ; 119:$ el10-26.

17. Proia RL, Hla T. Emerging biology of sphingosine-1-phosphate: its role in pathogenesis and therapy. J Clin Invest $2015 ; 125: 1379-87$.

18. Blaho VA, Chun J. Crystal clear? Lysophospholipid receptor structure insights and controversies. Trends Pharmacol Sci $2018 ; 39$ : 953-66.

19. Sarramegna V, Muller I, Milon A, Talmont F. Recombinant $G$ protein-coupled receptors from expression to renaturation: a challenge towards structure. Cell Mol Life Sci. 2006 ; 63 : 1149-64.

20. Schioth HB, Fredriksson R. The GRAFS classification system of G-protein coupled receptors in comparative perspective. Gen Comp Endocrinol 2005 ; 142 : 94-101.

21. Cuvillier 0 . Les récepteurs de la sphingosine 1-phosphate: de la biologie à la physiopathologie. Med Sci (Paris) $2012 ; 28: 951-7$.

22. Alexander SPH, Christopoulos A, Davenport AP, et al. The concise guide to pharmacology 2019/20: G protein-coupled receptors. BrJ Pharmacol 2019; 176 (suppl 1) : S21-141.

23. Liu Y, Wada R, Yamashita T, et al. Edg-1, the G protein-coupled receptor for sphingosine-1phosphate, is essential for vascular maturation. J Clin Invest 2000 ; 106: 951-61.

24. Schwab SR, Cyster JG. Finding a way out: lymphocyte egress from lymphoid organs. Nat Immunol $2007 ; 8: 1295-301$.
25. Healy LM, Antel JP. Sphingosine-1-phosphate receptors in the central nervous and immune systems. Curr Drug Targets 2016 ; 17 : 1841-50.

26. Talmont F, Mouledous L. Evaluation of commercial antibodies against human sphingosine-1-phosphate receptor 1. Naunyn Schmiedebergs Arch Pharmacol 2014 ; 387 : 427-31.

27. Talmont F, Mouledous L, Baranger M, et al. Development and characterization of sphingosine l-phosphate receptor 1 monoclonal antibody suitable for cell imaging and biochemical studies of endogenous receptors. PLoS One 2019; 14 : e0213203.

28. Talmont F, Mouledous L, Boue J, et al. Denatured G-protein coupled receptors as immunogens to generate highly specific antibodies. PLoS One $2012 ; 7$ : e46348.

29. Adada M, Canals D, Hannun YA, Obeid LM. Sphingosine-1-phosphate receptor 2. FEBS J $2013 ; 280: 6354-66$.

30. Seyedsadr MS, Weinmann 0 , Amorim A, et al. Inactivation of sphingosine1-phosphate receptor 2 (S1PR2) decreases demyelination and enhances remyelination in animal models of multiple sclerosis. Neurobiol Dis 2019 ; 124 : 189-201.

31. Cattoretti G, Mandelbaum J, Lee N, et al. Targeted disruption of the SIP2 sphingosine 1-phosphate receptor gene leads to diffuse large B-cell lymphoma formation. Cancer Res 2009 ; 69 : 8686-92.

32. Jaillard C, Harrison S, Stankoff B, et al. Edg8/S1P5: an oligodendroglial receptor with dual function on process retraction and cell survival.J Neurosci $2005 ; 25$ : 1459-69.

33. Van Doorn R, Lopes Pinheiro MA, Kooij G, et al. Sphingosine 1-phosphate receptor 5 mediates the immune quiescence of the human brain endothelial barrier. J Neuroinflammation $2012 ; 9$ : 133.

34. Graler MH, Goetzl $\varepsilon$ J. The immunosuppressant FTy720 down-regulates sphingosine 1-phosphate G-protein-coupled receptors. FASEB J 2004 ; 18: 551-3.

35. 0o ML, Thangada S, Wu MT, et al. Immunosuppressive and anti-angiogenic sphingosine 1-phosphate receptor-1 agonists induce ubiquitinylation and proteasomal degradation of the receptor. J Biol Chem 2007 ; 282 : 9082-9.

36. Huwiler A, Zangemeister-Wittke U. The sphingosine 1-phosphate receptor modulator fingolimod as a therapeutic agent: Recent findings and new perspectives. Pharmacol Ther 2018 ; $185: 34-49$.

37. Sanna MG, Vincent KP, Repetto $\varepsilon$, et al. Bitopic sphingosine 1-phosphate receptor 3 (SIP3) antagonist rescue from complete heart block: pharmacological and genetic evidence for direct S1P3 regulation of mouse cardiac conduction. Mol Pharmacol 2016 ; 89 : 176-86.

38. Kremer D, Gottle P, Flores-Rivera J, et al. Remyelination in multiple sclerosis: from concept to clinical trials. Curr Opin Neurol 2019 ; 32 : 378-84.

39. Sun L, Telles $\varepsilon$, Karl M, et al. Loss of HDACll ameliorates clinical symptoms in a multiple sclerosis mouse model. Life Sci Alliance 2018 ; 1 : e201800039.

40. Mi S, Miller RH, Lee $X$, et al. LINGO-1 negatively regulates myelination by oligodendrocytes. Nat Neurosci $2005 ; 8: 745-51$.

41. Cadavid D, Balcer L, Galetta $S$, et al. Safety and efficacy of opicinumab in acute optic neuritis (RENEW): a randomised, placebo-controlled, phase 2 trial. Lancet Neurol 2017 ; 16 : 189-99.

42. Cadavid D, Mellion M, Hupperts R, et al. Safety and efficacy of opicinumab in patients with relapsing multiple sclerosis (SYNERGY): a randomised, placebo-controlled, phase 2 trial. Lancet Neurol 2019; 18 : 845-56.

43. Green AJ, Gelfand JM, Cree BA, et al. Clemastine fumarate as a remyelinating therapy for multiple sclerosis (ReBUILD): a randomised, controlled, doubleblind, crossover trial. Lancet 2017 ; $390: 2481-9$.

44. Bordet R, Camu W, De Seze J, et al. Mechanism of action of slp receptor modulators in multiple sclerosis: the double requirement. Rev Neurol (Paris) 2019 ; Nov 19. pii: S0035-3787(18)30499-5.

45. Jackson SJ, Giovannoni G, Baker D. Fingolimod modulates microglial activation to augment markers of remyelination. J Neuroinflammation $2011 ; 8: 76$.

46. Szepanowski F, Derksen A, Steiner I, et al. Fingolimod promotes peripheral nerve regeneration via modulation of lysophospholipid signaling. J Neuroinflammation $2016 ; 13: 143$.

47. Kim HJ, Miron VE, Dukala D, et al. Neurobiological effects of sphingosine 1-phosphate receptor modulation in the cuprizone model. FASEB J 2011 ; $25: 1509-18$.

48. Gentile A, Musella A, Bullitta S, et al. Siponimod (BAF312) prevents synaptic neurodegeneration in experimental multiple sclerosis. J Neuroinflammation $2016 ; 13: 207$.

49. Sedel F, Papeix C, Bellanger A, et al. High doses of biotin in chronic progressive multiple sclerosis: a pilot study. Mult Scler Relat Disord 2015 ; $4: 159-69$. 


\section{RÉFÉRENCES}

50. Cuascut $F X$, Hutton GJ. Stem cell-based therapies for multiple sclerosis: current perspectives. Biomedicines $2019 ; 7$.

51. Burt RK, Balabanov R, Burman J, et al. Effect of nonmyeloablative hematopoietic stem cell transplantation vs continued disease-modifying therapy on disease progression in patients with relapsing-remitting multiple sclerosis: a randomized clinical trial. Jama $2019 ; 321$ : 165-74.

52. Kvistad SAS, Lehmann AK, Trovik LH, et al. Safety and efficacy of autologous hematopoietic stem cell transplantation for multiple sclerosis in Norway. Mult Scler 2019 : 1352458519893926.

53. Muraro PA, Pasquini M, Atkins HL, et al. Long-term outcomes after autologous hematopoietic stem cell transplantation for multiple sclerosis. JAMA Neurol $2017 ; 74: 459-69$.

54. Mansoor SR, Zabihi $\varepsilon$, Ghasemi-Kasman M. The potential use of mesenchymal stem cells for the treatment of multiple sclerosis. Life Sci $2019 ; 235$ : 116830.
55. Uccelli A, Laroni A, Brundin L, et al. Mesenchymal stem cells for multiple sclerosis (MESEMS): a randomized, double blind, cross-over phase I/II clinical trial with autologous mesenchymal stem cells for the therapy of multiple sclerosis. Trials $2019 ; 20: 263$.

56. Shroff $G$. A review on stem cell therapy for multiple sclerosis: special focus on human embryonic stem cells. Stem Cells Cloning 2018; 11 : 1-11.

\section{www.myobase.org}

Catalogue en ligne disponible gratuitement sur Internet publié par l'AFM-Téléthon.

Retrouvez facilement toutes les références bibliographiques sur les maladies neuromusculaires, les situations de handicap qu'elles génèrent et leurs aspects psychologiques.

Myobase donne un accès libre à $75 \%$ du fonds documentaire collecté depuis 1990, représentant plus de 40000 références spécifiques du domaine des maladies neuromusculaires.

$>$ articles de la littérature biomédicale et psycho-sociale

\section{$>$ livres, thèses}

$>$ guides d'associations et rapports institutionnels d'agences internationales

$>$ brèves en français, synthèses des articles médico-scientifiques internationaux les plus pertinents

\section{$>$ publications}

AFM-Téléthon destinées aux professionnels de santé ou aux personnes atteintes de maladie neuromusculaire et à leur entourage
UN OUTIL ERGONOMIQUE,
UNE INTERFACE BILINGUE

- Laissez-vous guider par les tutoriels

- Lancez une recherche et affinez votre sélection grâce aux filtres

\begin{tabular}{|c|c|}
\hline \multicolumn{2}{|c|}{ TOUT MYOBASE } \\
\hline \multicolumn{2}{|c|}{ Rechercher... } \\
\hline \multicolumn{2}{|c|}{ Recherche avancée } \\
\hline \multirow[t]{6}{*}{ Histo } & FILTRES \\
\hline & Type de document \\
\hline & D Article [3443] \\
\hline & Publication AFM [176] \\
\hline & $\square$ Thèse/Mémoire [107] \\
\hline & Brève [102] \\
\hline
\end{tabular}

- PUBLICATIONS AFM-Téléthon

○ BRĖVES

$\rightarrow$ DOCUMENTS DE SYNTHËSE

INSTITUT DES BIOTHÉRAPIES PUBLICATIONS

- Partagez les résultats de votre recherche
TIRÉS À PART

O. Cuvillier

\section{UN ACCÈS facile et simple}

Rechercher avec des opérateurs :

- guillemets pour une expression "maladie de pompe"

- + pour signifier ET, et retrouver tous les documents contenant les deux mots "fauteuil +électrique"

- - pour signifier NON et enlever le mot de la recherche:

"autonomie -établissement"
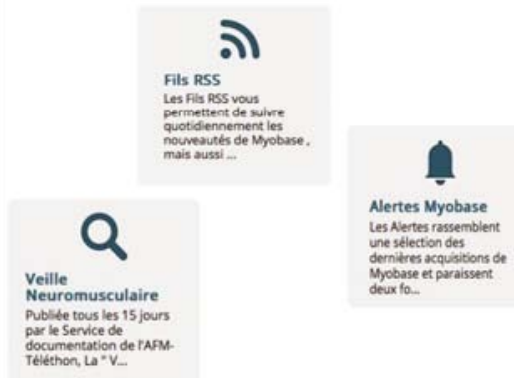

- Cliquez sur I'onglet thématique qui vous convient (haut de la page d'accueil)

- Créez vos alertes personnalisées en ouvrant un compte personnel

- Téléchargez la Veille Neuromusculaire

- Abonnez-vous aux flux RSS 